\title{
Can We Offer More to Patients with Multiple Sclerosis?
}

\author{
Highlights of a Satellite Symposium Presented at the 9th World Congress on Controversies in Neurology, \\ 26-28 March 2015, Budapest, Hungary
}

Expert Review by: Bart Van Wijmeersch, ${ }^{1}$ Celia Oreja-Guevara ${ }^{2}$ and Ron Milo ${ }^{3}$

1. Professor, Rehabilitation \& MS-Centre Overpelt, BIOMED, University Hasselt, Belgium; 2. Head of Clinical Research, Multiple Sclerosis Unit, Hospital Clinico San Carlos, Madrid, Spain; 3. Head, Department of Neurology, Barzilai Medical Center, Ashkelon, Faculty of Health Sciences, Ben-Gurion University of the Negev, Israel

\begin{abstract}
The recent approvals of both teriflunomide $\left(\right.$ Aubagio $^{\circledR}$ ) and alemtuzumab (Lemtrada ${ }^{\circledR}$ ) have meant that substantially more can now be offered to patients with multiple sclerosis (MS). In clinical trials, teriflunomide has shown consistent efficacy across patients with early disease (TOPIC study, $n=618$ ) and in patients with relapsing forms of MS (TEMSO, $n=1,088$ and TOWER studies $n=1,169$ ). Teriflunomide $14 \mathrm{mg} /$ day showed consistent efficacy in patients with varying levels of disease activity and is the only approved oral MS therapy that significantly delayed disability progression in two phase III clinical trials. The safety profile of teriflunomide now extends to 12 years and the data support its use as a platform agent in patients with relapsing MS (RMS). In other phase II and III clinical trials (CAMMS223, n=334, CARE-MS 1, n=581 and CARE-MS 2, n=840), alemtuzumab has demonstrated superior efficacy (clinical and magnetic resonance imaging [MRI]) than high-dose subcutaneous (S.c.) interferon beta (IFN $\beta$-1a). It has also shown improvement in pre-existing disability compared with IFN $\beta$-1a s.c. and sustained efficacy over 3-4 years despite no further therapy after the second administration in the majority of the patients. Alemtuzumab has a consistent, well-characterised safety profile, so adverse events can be identified and managed using a comprehensive safety monitoring and education programme. Both teriflunomide and alemtuzumab therefore have favourable benefitrisk profiles in patients with early and/or active RMS. Their efficacies constitute real advances in MS treatment and in regular clinical use are likely to effectively control disease and improve outcomes for many MS patients.
\end{abstract}

\section{Keywords}

Teriflunomide, alemtuzumab, efficacy, safety, multiple sclerosis, patient selection, treatment administration, treatment timing

\begin{abstract}
Disclosure: Bart Van Wijmeersch has received research and travel grants and honoraria for multiple sclerosis expert advice and speakers fees from Bayer-Schering, Biogen, Genzyme-Sanofi, Merck-Serono, Novartis and Teva. Celia Oreja-Guevara has received advisory board and consulting honoraria from Bayer, Biogen, Genzyme-Sanofi, Merck-Serono, Novartis and Teva. Ron Milo has received research grants, travel funding, speaker's honoraria or consultation fees from Bayer-Schering, Biogen Idec, Genzyme, Medison Pharma, Neopharm, Novartis, Merck-Serono and Teva.

Acknowledgements: Editorial assistance was provided by James Gilbart at Touch Medical Media, London and funded by Genzyme. This article reports the proceedings of a sponsored satellite symposium and as such has not been subject to the journal's usual peer-review process.

Open Access: This article is published under the Creative Commons Attribution Noncommercial License, which permits any non-commercial use, distribution, adaptation and reproduction provided the original author(S) and source are given appropriate credit.

Received: 1 July 2015 Published Online: 26 August 2015 Citation: European Neurological Review, 2015;10(2):139-47 http://doi.org/10.17925/ENR.2015.10.02.139 Correspondence: Ron Milo, Department of Neurology, Barzilai Medical Center, Ashkelon 78278, Israel E: rmilo@barzi.health.gov.il
\end{abstract}

Support: This article was supported by Genzyme (a Sanofi company).

Despite the availability of disease-modifying therapies (DMTs) in multiple sclerosis (MS) for over 2 decades, substantial unmet treatment needs have remained. From the patient perspective, there is a need for better tolerability, quality of life (QoL) benefits and customised treatment approaches that are based on disease prognosis and individual patient needs and risk- benefit ratio. From the healthcare provider perspective, there is a need for treatment approaches that address individual prognosis, optimised treatment outcomes in an increasingly complex treatment landscape and ability to achieve new treatment goals. ${ }^{1-11}$ The recent approvals of the oral treatment teriflunomide (Aubagio ${ }^{\circledR}$ ) and the intravenous (IV) monoclonal antibody (mAb) treatment alemtuzumab (Lemtrada ${ }^{\circledR}$ ) in MS have markedly changed the options available to neurologists and their ability to achieve these goals. This review considers how teriflunomide and alemtuzumab can fit into current and future treatment approaches to MS as discussed at a satellite symposium and a plenary session that were convened at the 9th Controversies in Neurology meeting at Budapest, Hungary, in March 2015.

\section{Teriflunomide - For Whom, When, How?}

Teriflunomide is a once-daily oral therapy that was approved for the treatment of relapsing forms of MS by the US Food and Drug Administration (FDA) in September 2012 and by the EU Commission in Europe in August 2013. The mode of action of teriflunomide in MS is not fully understood but it is known to selectively and reversibly inhibit dihydroorotate reductase, a key mitochondrial enzyme involved in de novo pyrimidine synthesis, which is required for the proliferation of activated lymphocytes. This results in fewer $B$ and $T$ cells crossing the blood-brain barrier. Teriflunomide also reduces the ability of activated $B$ and $T$ cells to participate in the damaging immune attack on the central nervous system and has other modes of action. ${ }^{12}$ These actions are quite specific and preserve adaptive 


\section{Table 1: Design of the Phase III TEMSO and TOWER studies of Oral Teriflunomide in Multiple Sclerosis}

\begin{tabular}{|c|c|c|}
\hline & TEMSO & TOWER \\
\hline Study design & \multicolumn{2}{|c|}{$\begin{array}{l}\text { Multicentre, multinational, randomised, double-blind, } \\
\text { parallel-arm, placebo-controlled }\end{array}$} \\
\hline Patients (randomised), $n$ & 1,088 & 1,169 \\
\hline Study duration & 2 years & $\begin{array}{l}\text { Fixed end for all patients } \\
48 \text { weeks from last patient } \\
\text { randomised }\end{array}$ \\
\hline Patient population & \multicolumn{2}{|c|}{$\begin{array}{l}\left.\text { Patients with RMS (McDonald criteria }{ }^{3,4}\right) \\
\text { Aged } 18-55 \text { years } \\
\text { EDSS score } \leq 5.5 \text { at screening } \geq 2 \text { relapses within } 2 \text { years } \\
\text { or } \geq 1 \text { relapse within } 1 \text { year before randomisation }\end{array}$} \\
\hline Treatment arms & \multicolumn{2}{|c|}{$\begin{array}{l}\text { Once-daily, oral } \\
\text { Placebo : Teriflunomide } 7 \mathrm{mg} \text { : Teriflunomide } 14 \mathrm{mg}\end{array}$} \\
\hline Primary outcome & \multicolumn{2}{|c|}{ ARR } \\
\hline Secondary outcomes & $\begin{array}{l}\text { Key: Disability progression } \\
\text { (confirmed over } 12 \text { weeks) } \\
\text { Additional: MRI measures, } \\
\text { Safety }\end{array}$ & $\begin{array}{l}\text { Key: Disability progression } \\
\text { (confirmed over } 12 \text { weeks) } \\
\text { Additional: Safety }\end{array}$ \\
\hline
\end{tabular}

$\overline{A R R}=$ annualised relapse rate; $E D S S=$ Expanded Disability Status Scale;

$M R I=$ magnetic resonance imaging; $R M S$ = relapsing multiple sclerosis:

TEMSO = Randomized Trial of Oral Teriflunomide for Relapsing Multiple Sclerosis

Patients study; TOWER = Teriflunomide Oral in People with Relapsing Multiple Sclerosis Study. Sources: O'Connor et al. 2011, ${ }^{16}$ Confavreux et al. 2014, ${ }^{17}$ MCDonald et al. 2001, ${ }^{57}$ Polman et al. 2005.58

immunity to infectious pathogens, which is an advantage over some other immunomodulating agents used in MS. ${ }^{13,14}$ Teriflunomide is not cytotoxic and does not deplete lymphocytes, so can be described as an immunomodulatory agent.

\section{Clinical Trial Evidence}

The clinical development programme of teriflunomide in MS began in 2001 and, to date, has demonstrated favourable efficacy and safety findings in extensive phase II and III studies. For some of these, extension phases are continuing:

- Phase II teriflunomide versus placebo in patients with relapsingremitting MS (RRMS) or secondary progressive MS (SPMS) with relapses ( $n=179$ ) randomised controlled trial (RCT) with an ongoing open-label extension (147 entered the extension study). ${ }^{15}$

- Phase III randomised trial of oral teriflunomide for relapsing MS (RMS) patients (TEMSO) $(n=1,088) ;$ teriflunomide versus placebo RCT with an ongoing open-label extension. ${ }^{16}$

- Phase III teriflunomide oral in people with RMS (TOWER) $(n=1,169)$; teriflunomide versus placebo RCT with an ongoing blinded extension. ${ }^{17}$

- Phase III teriflunomide versus subcutaneous (s.c.) interferon beta1a (IFN $\beta$-1a) $(44 \mu g)$ in patients with RRMS (TENERE) ( $n=324)$ RCT with an ongoing open-label extension. ${ }^{18}$

- Phase III teriflunomide versus placebo in patients with the first clinical symptom of MS (TOPIC) $(n=618)$ teriflunomide versus placebo with an open-label extension. ${ }^{19}$

- Phase III study to investigate the immune response to influenza vaccine in patients with MS receiving teriflunomide doses or IFN $\beta$ 1a (TERIVA) $(n=128) .{ }^{20}$

The designs of the large-scale, pivotal TEMSO ${ }^{16}$ and TOWER ${ }^{17}$ studies are summarised in Table 1. The populations had similar demographics and disease history; the mean patient ages were 37.9 years in both and $72 \%$ and $71 \%$ of patients, respectively, were female. The mean times since MS diagnosis were 8.7 and 8.0 years and the mean number of relapses within the previous two years were 2.2 and 2.1. These studies included mostly patients with RRMS (91.5\% and $97.5 \%$ ) but also included some with secondary progressive MS (4.7 \% and $0.8 \%$ ) and progressive relapsing MS (3.9 \% and $1.7 \%)$. The mean baseline Expanded Disability Status Scale (EDSS) scores were 2.7 in both studies. ${ }^{21}$

Both the TEMSO and TOWER studies showed notable improvements in primary and secondary MS endpoints for teriflunomide compared with placebo. Both these studies showed consistent and reproducible results. After 2 years, the annualised relapse rate (ARR) was reduced by $32 \%$ and $31 \%$ in patients treated with $14 \mathrm{mg}$ and $7 \mathrm{mg}$ teriflunomide, respectively, in the TEMSO study ( $p<0.001$ for both comparisons). ${ }^{16}$ There were also $30 \%$ and $24 \%$ relative risk reductions in confirmed (>12 week) disability accumulation for patients treated with $14 \mathrm{mg}$ or $7 \mathrm{mg}$ teriflunomide, respectively ( $\mathrm{p}=0.028$ and $p=0.084$, respectively). The TEMSO study included magnetic resonance imaging (MRI) assessments that showed a marked effect of teriflunomide treatment. In particular, after 108 weeks there was a mean dose response on total lesion volume (-39.4\%; $p=0.03$ for $7 \mathrm{mg}$ and $-67.4 \%, p<0.001$ for 14 $\mathrm{mg})$. At this time-point relative to placebo, mean white matter volume was $83.0 \%$ in the $7 \mathrm{mg}$ group ( $\mathrm{p}=0.0609$ ) and $164.3 \%$ in the $14 \mathrm{mg}$ group $(p=0.0002) .{ }^{22}$ This indicates pathological improvement during teriflunomide treatment compared with placebo, which was significant for the $14 \mathrm{mg}$ dose.

In the TOWER study, after 2.5 years treatment, ARR was reduced by $36 \%$ and $22 \%$ in patients treated with $14 \mathrm{mg}$ or $7 \mathrm{mg}$ teriflunomide, respectively, compared with placebo, $\left(p<0.0001\right.$ and $p=0.0183$, respectively). ${ }^{17}$ There were also $32 \%$ and $5 \%$ relative risk reductions for confirmed (>12 week) disability accumulation for patients treated with $14 \mathrm{mg}$ or $7 \mathrm{mg}$ teriflunomide, respectively ( $\mathrm{p}=0.044$ and $\mathrm{p}=0.762$, respectively)

In the TERIVA study, patients with RRMS were assigned to receive $14 \mathrm{mg}(\mathrm{n}=41)$ or $7 \mathrm{mg}(\mathrm{N}=41)$ teriflunomide/day or s.C. IFN $\beta-1 a$ $44 \mu \mathrm{g} 3 \times$ weekly $(\mathrm{n}=46)$ for at least 6 months prior to administration of influenza vaccine containing H1N1, H3N2 and B strains. After 28 days, anti-H1N1 titres $\geq 40$ were achieved in most patients $(97.5 \%$, $97.4 \%$ and $97.7 \%$, respectively). Anti-H2N3 titres $\geq 40$ were achieved in slightly lower proportions ( $90.0 \%, 76.9 \%$ and $90.7 \%$, respectively). This study indicated that teriflunomide does not adversely affect the ability of treated patients to mount an immune response against influenza and that immune competence to this vaccine during such treatment was similar to that of IFN $\beta-1$.

In addition to the pivotal studies, other studies on teriflunomide treatment of MS have been completed or are in progress. One phase I study showed the limited effect of teriflunomide on responses to rabies vaccination. ${ }^{23}$ Healthy subjects were given teriflunomide $7 \mathrm{mg}$ once daily for 5 days followed by $14 \mathrm{mg}$ once daily for 25 days $(n=23)$ or placebo (for 30 days; $n=23$ ). They were also given single intramuscular injections of rabies vaccine on days 5,12 and 31 . Antirabies geometric mean titres were lower in teriflunomide-treated subjects compared with placebo (relative titres: $0.75,0.63$ and 0.53 at days 19, 31 and 38, respectively). The results showed, however, that anti-rabies antibody levels above the $0.5 \mathrm{IU} / \mathrm{ml}$ threshold for seroprotection were induced in all subjects. 
The effect of teriflunomide on the immune system, in particular, the effect on blood lymphocyte subsets, is being further investigated in the Teri-Dynamic study that includes 48 patients with RRMS. ${ }^{24}$ In addition, the benefits and safety of teriflunomide in routine clinical practice are being investigated in an open-label, single-arm, noncomparative study (TERIPRO, $n=\sim 1,000$ ). ${ }^{25}$ An ongoing phase III study is investigating the efficacy, safety and pharmacokinetics of teriflunomide versus placebo in children and adolescents with RMS ( $n=165$, TERIKIDS). ${ }^{26}$ Teriflunomide evaluation is also continuing in a series of ongoing or planned phase IV post-marketing studies.

A more recent ad hoc pooled analysis of the combined TEMSO and TOWER studies showed that in the high-activity disease subgroup $(\geq 2$ relapses in the year before study entry) after treatment durations of up to 152 weeks, the $14 \mathrm{mg}$ dose produced a significant mean reduction of $34 \%$ in the ARR compared with placebo ( $p=0.001) .{ }^{27}$ In addition, the analysis showed a significant reduction of $46 \%$ (hazard ratio 0.543 ) for 12-week confirmed disability progression for the $14 \mathrm{mg}$ dose compared with placebo $(p=0.004)$ (see Figure 1). This indicates a notable benefit of teriflunomide treatment in MS.

In the ad hoc TEMSO/TOWER pooled analysis, placebo-treated patients had ARRs that were greater among those who had received $>1$ prior DMT than those who had received one prior DMT whose ARRs were greater than those who had received no prior DMT. For patients receiving $14 \mathrm{mg}$ teriflunomide and who had received $>1$ prior DMT, 1 prior DMT or no prior treatment reductions in ARR versus placebo were $46.7 \%, 27.7 \%$ and $35.9 \%$, respectively. For patients receiving $7 \mathrm{mg}$ teriflunomide, these reductions were $41.6 \%, 16.4 \%$ and $30.2 \%$, respectively. ${ }^{28}$ This indicates that teriflunomide treatment provides better results in patients who had received multiple previous DMTs. Both the $7 \mathrm{mg}$ and $14 \mathrm{mg}$ doses of teriflunomide were also shown to significantly reduce relapse severity-related outcomes compared with placebo. ${ }^{29}$ These included relapses causing increases in EDSS/functional systems score (FSS) over 30 days ( $p<0.001$ for both doses), relapses with sequelae (reported by investigator) $(p=0.046 ; p<0.001)$, relapse leading to hospitalisation ( $p<0.001 ; p=0.020)$ and relapses requiring IV corticosteroids ( $p<0.001$ for both doses).

In addition, the TEMSO/TOWER pooled analysis showed that healthrelated QoL (HRQOL) measures (Short Form [SF]-36 physical and mental component scores and utility index) were significantly worsened due to relapses with sequelae $(p<0.0001-0.005)$ or by relapses requiring hospitalisation $(p<0.0001) .{ }^{30}$

In the TOPIC study, patients were treated within 1.8-1.9 months of their first clinical event suggestive of MS. After 108 weeks there was a $43 \%$ reduction in the relative risk of conversion to clinically definite MS for $14 \mathrm{mg}$ teriflunomide dose versus placebo $(p=0.0087)$ and a $37 \%$ reduction for $7 \mathrm{mg}$ teriflunomide $(p=0.0271){ }^{19}$ In addition, at the same time-point there was a $35 \%$ reduction in the risk of a new clinical relapse or MRI lesion for the $14 \mathrm{mg}$ dose $(p=0.0003)$ and a $31 \%$ reduction ( $p=0.002)$ for the $7 \mathrm{mg}$ dose. This study also showed a $59 \%$ reduction in the number of gadolinium (Gd)-enhancing lesions per scan for the $14 \mathrm{mg}$ dose versus placebo $(p=0.0008)$ and a significant change from baseline in total lesion volume at 108 weeks versus placebo $(p=0.0374)$. These findings indicate that early treatment with teriflunomide in MS provides significant improvements in both clinical and MRI measures.

\section{Figure 1: A. Annualised Relapse Rate and B. Confirmed Disability Progression in a Combined Analysis of the TEMSO and TOWER Studies of Teriflunomide in Multiple Sclerosis}

A



B



HRR = hazard ratio reduction; TEMSO = The Teriflunomide for Relapsing Multiple Sclerosis Patients study; TOWER = The Teriflunomide Oral in People with Relapsing Multiple Sclerosis study. Source: Kappos et al. 2013. ${ }^{27}$

Teriflunomide has a favourable safety and tolerability profile that has been established during the pivotal studies. In the TEMSO and TOWER studies, there were no new or unexpected adverse events (AES) or serious AES (SAES). ${ }^{15,31}$ AEs that were more common with teriflunomide compared with placebo were: diarrhoea, elevated alanine aminotransferase (ALT) level, nausea, headache, elevated blood pressure and hair thinning. The incidence of SAES was limited and similar to that of placebo. A more recent pooled analysis of data from the phase II, TEMSO, and the TOWER and TOPIC studies showed that in the teriflunomide $14 \mathrm{mg}, 7 \mathrm{mg}$ and placebo groups hair thinning occurred in $13.9 \%, 10.0 \%$ and $5.1 \%$, respectively. ${ }^{15-17,21,31-33}$ Nausea occurred in $10.7 \%, 8.0 \%$ and $7.2 \%$; diarrhoea occurred in $13.6 \%, 13.2 \%$ and $7.6 \%$ and hepatic events occurred in $21.5 \%$, $19.8 \%$ and $15.2 \%$, respectively. There was little difference in serious infection incidence and no difference in benign/malignant tumours. Reductions in neutrophil and lymphocyte counts with teriflunomide were small in magnitude but greater for the $14 \mathrm{mg}$ dose than the $7 \mathrm{mg}$ dose or placebo..$^{16,17,33}$ As a result of safety findings, patients receiving teriflunomide must be monitored for ALT levels, blood pressure and total blood count. Teriflunomide was shown to be embryotoxic and teratogenic in rats and rabbits given doses in the human therapeutic range. ${ }^{34}$ Female patients receiving teriflunomide therefore must be checked for pregnancy status. 


\section{Multiple Sclerosis}

The TENERE study was a superiority trial involving a head-to-head comparison of teriflunomide doses with IFN $\beta-1 a$. This study did not show a significant difference in efficacy between teriflunomide and IFN $\beta$-1a. Time-to-treatment failure was comparable between teriflunomide $7 \mathrm{mg} /$ day, $14 \mathrm{mg} /$ day and IFN $\beta$-1a $44 \mu \mathrm{g}$ (three $\mathrm{x}$ weekly) over 96 weeks..$^{18}$ Patients reported less fatigue with both teriflunomide doses (significant for $7 \mathrm{mg}$ dose; $\mathrm{p}=0.03$ ). In a Treatment Satisfaction Questionnaire for Medication, scores in domains of global satisfaction, side effects and convenience were significantly improved with both doses of teriflunomide compared with IFN $\beta$-1a (global satisfaction: $p=0.02$ for both doses, side effects: $p<0.01$ for both doses and improved convenience: $p<0.01$ for both doses). ${ }^{18,35}$ AEs in this study that were slightly more frequent with teriflunomide $7 \mathrm{mg}$ and $14 \mathrm{mg} /$ day than IFN $\beta$-1a were: nasopharyngitis (26\%, $20 \%$ and $18 \%$, respectively), diarrhoea ( $23 \%, 21 \%$ and $8 \%$ ), hair thinning ( $6 \%, 20 \%$ and $1 \%$ ), paraesthesia (13\%, $10 \%$ and $8 \%$ ) and back pain $\left(9 \%, 10 \%\right.$ and $7 \%$ ). ${ }^{36}$ AEs that were less frequent with teriflunomide $7 \mathrm{mg}$ and $14 \mathrm{mg} /$ day than IFN $\beta$-1a were: flu-like symptoms (4 \%, $3 \%$ and $54 \%$ ), increased ALT levels ( $11 \%, 10 \%$ and $31 \%$ ) and headache $(21 \%, 16 \%$ and $26 \%)$. The majority of these ALT elevations were $\leq$ three $x$ upper limits of normal (ULN) and occurred in the first few months of treatment. All serious ALT elevations were asymptomatic and reversible.

There are currently no biomarkers predictive of response to teriflunomide - such a development would be highly valuable in the development of personalised medicine approaches in MS. Diligent and continual monitoring of patients receiving teriflunomide and review of efficacy (including 6-monthly MRI) in each case remains the optimum strategy.

\section{Real-world Evidence}

While clinical trial findings provide strong evidence supporting the use of teriflunomide in MS, real-world clinical examples help indicate that patients are suitable candidates for the drug in routine clinical practice, when and how it should be given. The following cases examples provide some insight.

\section{Case Example 1}

A 31-year-old female medical secretary with two children had right optic neuritis (ON) in 2012. Neurological examination was normal and the ON resolved rapidly and completely after intravenous methyl prednisolone (IVMP). No MRI was performed at this point. In 2014, she reported significant weakness of the left lower limb and urinary urgency. Neurological examination showed a left Babinski sign, slight hypertonia in the lower limbs and a discrete hypopallesthesia (decreased sensitivity to vibration). She showed slight improvement after IVMP.

After 2 months she showed persistent weakness of the left leg, difficulty walking rapidly and rare urinary urgency. General examination was normal, EDSS was 2.0 (pyramidal 2, bladder 1). The diagnosis was clinically definite MS based on two relapses during previous 2 years. MRI scans of this patient revealed a slight load of periventricular, subcortical and right pinocular lesions and several Gd-enhancing lesions at various locations including the spinal cord. After discussions with neurologists and the patient a decision was made to treat her with teriflunomide $14 \mathrm{mg}$ /day with vitamin D supplementation and contraception.

\section{Case Example 2}

A 35-year-old male of Northwest African origin reported blurred vision in the left eye of 2 weeks duration. One week later he experienced disabling paraesthesias in the right hand and significant weakness of the left leg. Neurological examination showed a right pyramidal syndrome, an internuclear ophthalmoplegia and a moderate reduction in sensitivity to vibration in the lower limbs. General examination was normal, cerebrospinal fluid (CSF) showed lymphocyte counts of $14 / \mathrm{ml}$ and oligoclonal bands. Brain MRI scan showed multiple supra- and infra-tentorial lesions typical of MS including 3 Gd-enhancing periventricular lesions. The patient was treated with IVMP for 3 days. The symptoms were indicative of clinically isolated syndrome (CIS) and resolved completely after a few days. A diagnosis of definite MS was made according to the 2010 McDonald criteria. Due to several poor prognostic factors (gender, ethnic origin, polyregional attack, pyramidal and brainstem involvement, high brain MRI lesion load, Gd enhancement, OCB in the CSF) and the need to treat as soon as possible, it was decided to give the patient $14 \mathrm{mg} /$ day teriflunomide.

\section{Case Example 3}

A 37-year-old female patient who had one child reported dizziness, left side paraesthesias and fatigue in October 2012, which resolved spontaneously. In December 2012 she had right peripheral facial palsy. In January 2013 an MRI scan showed lesions in the cerebral white matter (see Figure 2) compatible with demyelination and CSF showed oligoclonal bands. Treatment with IVMP was given and a diagnosis of MS was made.

In February 2013 weekly treatment with intramuscular IFN $\beta$-1a was initiated but this was stopped in May 2013 due to a persistent flulike symptoms and fatigue. In May 2014 she reported weakness in the lower limbs and increased fatigue. She was again treated with IVMP. An MRI scan showed slight increase in lesion load. Alternative immunomodulatory treatment was rejected by the patient due to fear of injections and AES.

In November 2014 she showed increased weakness in lower limbs, dizziness, significant reduction in vibration sensation in the lower limbs and urinary urgency. An MRI scan in January 2015 showed a markedly increased lesion load with active Gd-enhancing lesions (see Figure 2). The EDSS of the patient was 3.5 (pyramidal 3, sensory 2 , bladder 2, with slight cognitive decline). For this patient, a decision was taken to treat with teriflunomide $14 \mathrm{mg}$ /day and she was well at the time of the presentation.

These cases indicate that teriflunomide may be a viable option for treating MS at differing stages, particularly for patients with mild to moderate relapsing MS of a few years duration or in newly diagnosed cases or CIS. These cases also emphasise that commencing teriflunomide treatment can be suitable in cases of CIS and can help avoid adverse event risks associated with other oral MS treatments.

\section{Alemtuzumab - For Whom, When, How?}

Alemtuzumab is a humanised mAb treatment with a mode of action in MS that has not been fully elucidated but it is known to selectively target CD52 antigens on B- and T-lymphocytes, thus depleting these cells. ${ }^{37}$ This action effectively inhibits the inflammatory/neurodegenerative 


\section{Figure 2: Magnetic Resonance Imaging Scans of a Multiple Sclerosis Patient with Increasing Amount of Brain Lesions}


Source: Scans provided by Patrick Vermersch.

processes that are characteristic of MS. The treatment is given as a course of IV infusions (12 $\mathrm{mg}$ /day for 5 days) then a further course after 12 months ( $12 \mathrm{mg} /$ day for 3 days). Dosing appears to have only a minimal and transient effect on innate immunity (populations of $B$ and $T$ cells); counts of neutrophils, monocytes, basophils and eosinophils show little or no change in the months after a course of infusions and this minimises vulnerability to infection. Following alemtuzumab administration, its blood levels become undetectable after 1 month. However, there is a change in the number and proportions of certain Iymphocyte subsets, including a relative increase of $T$ regulatory (Treg) and memory $T$ cells (CD4 ${ }^{+}$CD45RA ${ }^{-4}$ ) compared with the overall T-cell population..$^{37-41}$ This sustained change in immune balance is a possible explanation for the long-lasting effects of alemtuzumab and its efficacy.

\section{Clinical Trial Evidence}

The clinical development programme consisted of three major trials (combined populations $n=1,755$ ) and a combined ongoing extension phase. The CARE-MS I and II trials were unusual for pivotal studies in being head-to-head designs rather than placebo-controlled. The active comparator in both trials was high-dose IFN $\beta 1$ 1a s.c. The primary endpoints in each case were ARR and time to 6-month sustained accumulation of disability (SAD): $38,42,43$

- Phase II CAMMS223 3-year study, alemtuzumab versus IFNß1a s.c. in treatment-naïve RRMS ( $n=334)$.
Figure 3: Improvement of Pre-existing Disability in Patients who Relapsed on Prior Therapy in the CARE-MS II Trial. A. 6-month Sustained Reduction In Disability B. EDSS
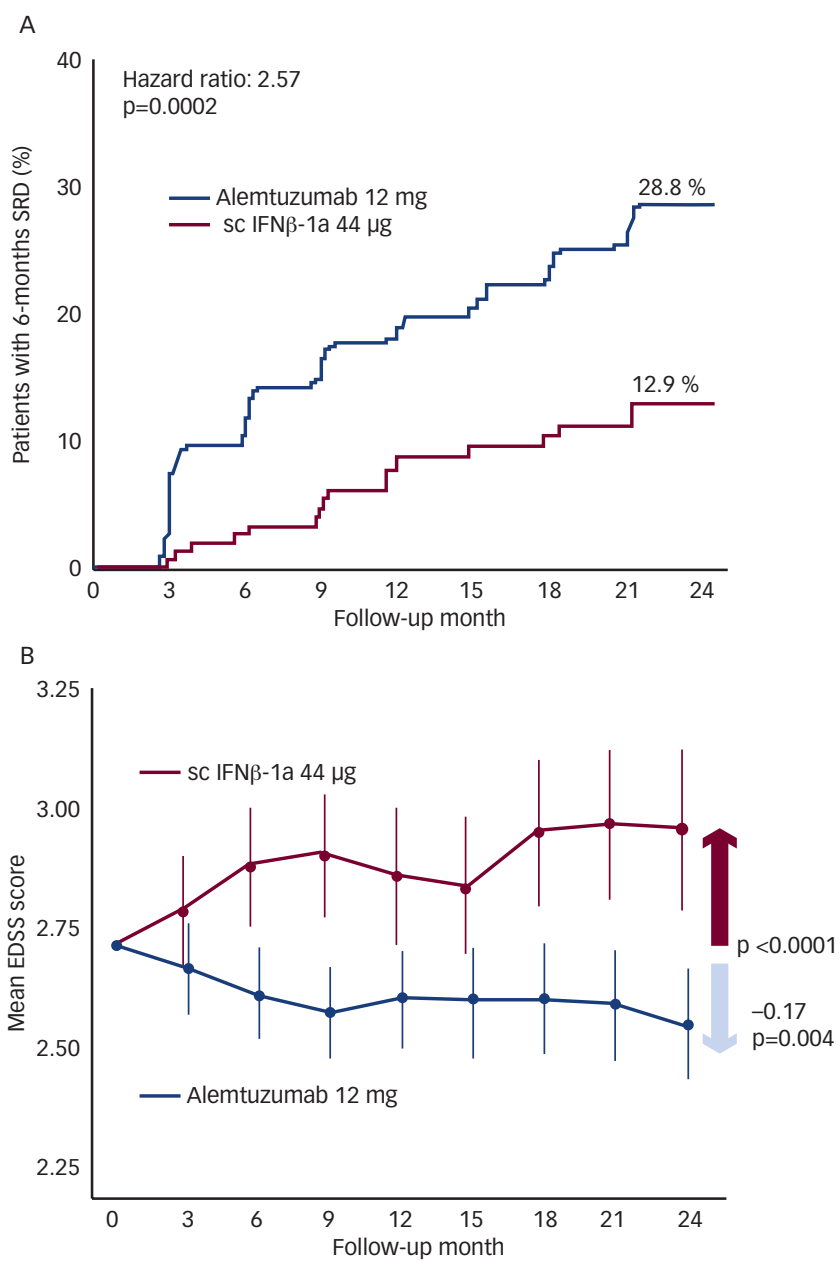

EDSS = Expanded Disability Status Scale; IFN $\beta=$ interferon beta; $S C=$ subcutaneous; SRD = sustained reduction in disability. Source: Cohen et al. 2012, ${ }^{62}$ Coles et al. 2012.38

- Phase III CARE-MS I, 2-year study alemtuzumab versus IFN $\beta 1$ 1a s.c. in treatment-naïve RRMS $(n=581)$.

- Phase III CARE-MS II, a 2-year study of alemtuzumab versus IFN $\beta 1$ a S.C. in RRMS patients who relapsed on prior therapy $(n=840)$.

- CARE-MS extension (ongoing, $n=1,322$ ) open to eligible patients from all three trials.

After 2 years, the CARE-MS I and II trials, reported $55 \%$ and $49 \%$ reductions in ARR for alemtuzumab compared with IFN $\beta$-1a s.c. ( $p>0.0001$ for both). In addition, $50 \%$ and $52 \%$ reductions in SAD were observed for alemtuzumab compared with IFN $\beta$-1a s.c. ( $p>0.0001$ for both). ${ }^{38,42}$ Similar efficacy was seen in both treatment-naïve and patients who had relapsed on prior therapy. Alemtuzumab also improved measures of disability in many patients. In the CARE-MS II trial, after 24 months, alemtuzumab-treated patients were more than twice as likely to show 6-month sustained reduction in disability (6-month SRD) compared with IFN $\beta$-1a s.c. (28.8\% versus $12.9 \% ; p=0.0002$ ). Furthermore, there was a mean increase of 0.24 points in the EDSS score for IFN $\beta$-1a s.c. but a mean decrease of 0.17 points for alemtuzumab $(p<0.0001)$ (see Figure 3 ). In addition, both the CARE-MS I and II trials showed significant improvements in measures of QoL for alemtuzumab versus IFN $\beta$-1a. 


\section{Figure 4: Brain Volume Loss Over 3 Years of Treatment with Alemtuzumab Compared with Subcutaneous IFN $\beta-1 a$ in A. the CARE-MS I and B. the CARE MS II Studies}
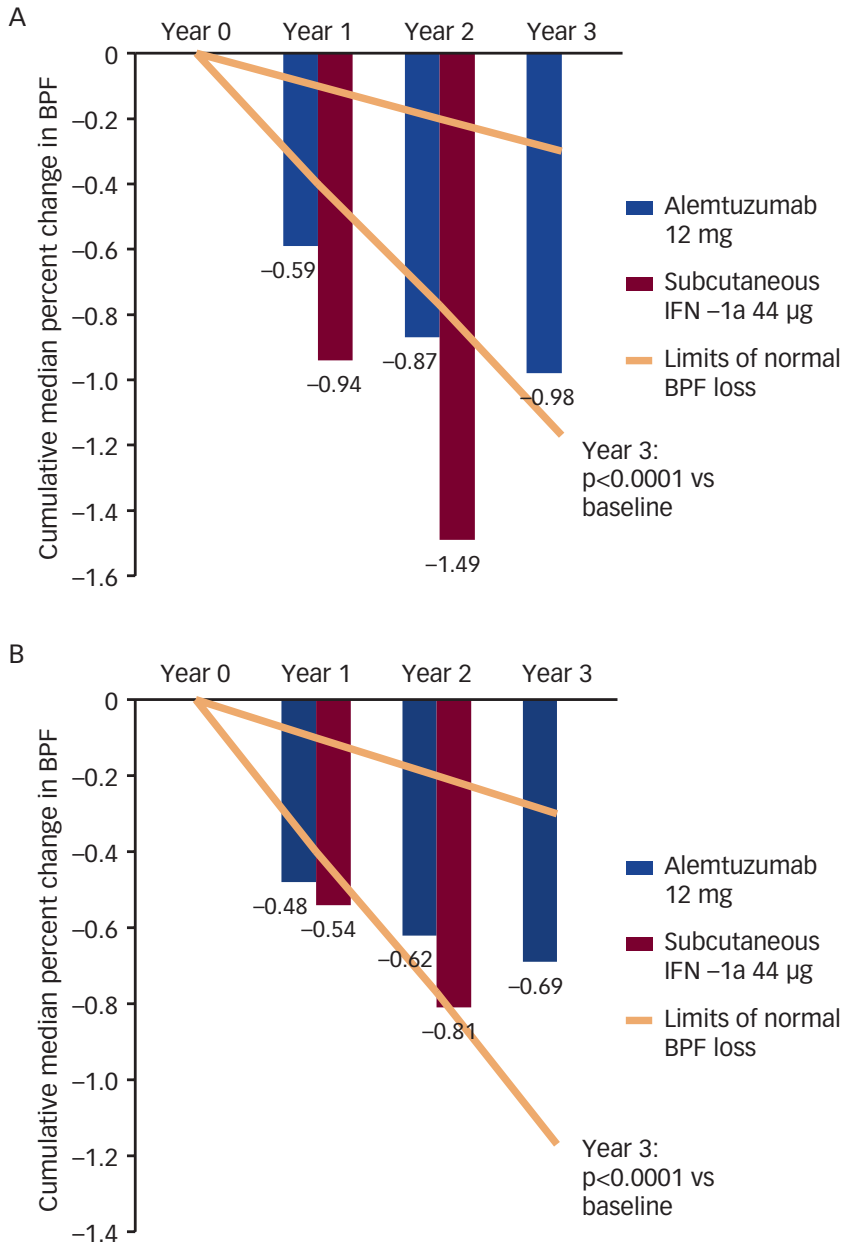

The area between the yellow lines represents the expected range of brain volume (BPF) loss in healthy people over a 3-year period. ${ }^{5,6} \mathrm{BPF}=$ brain parenchymal fraction; IFN $\beta=$ interferon beta. Source: Arnold et al. 2012; Arnold et al. 2014 Arnold et al. 2014.48-50

This was emphasised by Functional Assessment of Multiple Sclerosis (FAMS) scores in the CARE-MS I trial, which showed significantly greater improvement for alemtuzumab compared with IFN $\beta-1 a$ ( $p<0.05$ at months 6, 12, 18 and 24). In the CARE-MS II trial, FAMS scores were also significantly improved for alemtuzumab compared with IFN $\beta-1 a(p<0.005$ at months 6 and 12 and $p<0.0001$ at month 18). ${ }^{44,45}$

Results from the CARE-MS extension study show the long and durable efficacy of alemtuzumab treatment. Approximately $70 \%$ of treated patients received no additional alemtuzumab treatment courses through year 4 and less than $5 \%$ received another DMT during year 4 of the extension. ${ }^{46,47}$ Relapse rates showed continued control with alemtuzumab - in patients from the CARE-MS I trial ARRs were 0.19 and 0.14 /year for years 3 and 4 , respectively. In patients from the CARE-MS II trial,ARRs were 0.22 and 0.23 for years 3 and 4 , respectively. At the end of the randomised treatment period, the proportion with 6-month sustained reduction in disability was $29 \%$ rising to $35 \%$ after year 3 and $41 \%$ after year 4 . Most other patients remained stable with only a minority showing any worsening. In addition, EDSS remained stable or improved in the majority of treatment naive/ relapsing on prior treatment patients up to the end of year 4 . Over 4 years in the CARE-MS I study, disability improved or remained stable in $66.2 \%$ and worsened in $33.8 \% .{ }^{46}$ Over the same time period in the CARE-MS II study, disability improved or remained stable in $73.5 \%$ and worsened in $26.5 \%$.

Between the end of the 2-year randomised study periods and the end of the extension phase, patients in both the CARE-MS I and II studies showed slight increases in proportions with $\mathrm{T} 1$ hypointense lesions (22.2 \% and $22.7 \%$ increasing to $27.7 \%$ and $31.0 \%$, respectively) and $\mathrm{T} 2$ new/enlarging lesions ( $6.7 \%$ and $7.2 \%$ increasing to $10.8 \%$ and $12.5 \%$, respectively). In the CARE-MS I and II studies, the proportions of patients who received alemtuzumab and had Gd-enhancing lesions was markedly decreased after 3 years compared with baseline (46\% and $42 \%$ decreasing to $9.8 \%$ and $13.5 \%) .38,42,48$

The CARE-MS extension study has also highlighted the slowing of brain volume loss in the alemtuzumab patients arm. In the CARE-MS I trial, there was an MRI-determined cumulative median reduction in brain parenchymal fraction of $-0.98 \%$ ( $p>0.0001$ versus baseline) compared with $-1.49 \%$ for IFN $\beta-1$ a s.c over 3 years. ${ }^{48-50}$ In the CARE-MS I| trial, these mean brain parenchymal reductions were $-0.69 \%$ ( $p>0.0001$ versus baseline) compared with $-0.81 \%$ for IFN $\beta$-1a s.c .over 3 years. Brain volume loss for alemtuzumab-treated patients was in the normal range in both studies and showed signs of flattening compared with IFN $\beta$-1a s.c. (see Figure 4).

\section{Real-world Decisions on Who to Treat with Alemtuzumab, When and How?}

Alemtuzumab is indicated for adult patients with RRMS with active disease defined by clinical or imaging features. It can be used as first-line induction treatment to induce rapid remission in patients with highly-active disease and poor prognosis before switching to another maintenance therapy. Alemtuzumab can also be used as second-line treatment as part of an 'escalation strategy' in highly active disease after failure of first-line therapies: such patients require urgent intervention with a high efficacy treatment to prevent further damage. Alemtuzumab can also be used as a third-line treatment for 'breakthrough disease' when there is continuous disease activity despite second-line treatment with agents such as natalizumab or fingolimod.

\section{Case Example 1 - First-line treatment}

A 38-year-old female patient who had two relapses approximately 6 months apart - the second relapse was a left leg paresis, had a worsening EDSS score to 3.0 and a large new Gd-enhancing MRI lesion. She was given IVMP to treat the relapse followed by two courses of alemtuzumab. Her EDSS improved from 2.5, after MPIV, to 2.0 and remained stable for 3 years. Subsequent 6-monthly MRI scans showed no increase in T2 lesions and the Gd-enhancing lesion disappeared and did not develop into a black hole. Analysis of sequential MRI scans over 2 years showed that there had been an annualised brain volume loss of $1.48 \%$ during the 6 months prior to alemtuzumab treatment, which is consistent with the disease process. During the 18 months after the first alemtuzumab treatment the total percentage of brain volume loss was $0.4 \%$, which is within the normal range. There was little or no change in $\mathrm{T} 2$ lesion volume over the same period. 
Table 2: Risks Identified during the Alemtuzumab Clinical Development Programme

\begin{tabular}{|c|c|c|}
\hline Identified Risk & Rate in Alemtuzumab-treated Patients & Notes \\
\hline \multicolumn{3}{|l|}{ Autoimmune Disorders } \\
\hline $\begin{array}{l}\text { Immune thrombocytopenic } \\
\text { purpura }\end{array}$ & $\begin{array}{l}\sim 1 \%(1 \text { fatality prior to implementation of } \\
\text { monitoring programme })^{53}\end{array}$ & $\begin{array}{l}\text { Onset occurred 14-36 months after first exposure. Most cases responded to first- } \\
\text { line medical therapy }{ }^{53}\end{array}$ \\
\hline Nephropathies & $\begin{array}{l}0.3 \% \text { (anti-glomerular basement } \\
\text { membrane; } n=2 \text { ) }\end{array}$ & $\begin{array}{l}\text { Occurred within } 39 \text { months after last administration. Responded to } \\
\text { timely medical treatment and did not develop permanent kidney failure }{ }^{60}\end{array}$ \\
\hline $\begin{array}{l}\text { Thyroid disorders } \\
\text { (Hypo-/hyper-) }\end{array}$ & $\sim 36 \%$ (serious, $1 \%)^{53}$ & $\begin{array}{l}\text { Onset occurred 6-61 months after first alemtuzumab exposure; peaked in year } 3 \\
\text { and declined thereafter. }{ }^{61} \text { Mostly mild to moderate, managed with conventional } \\
\text { medical therapy; however, some patients required surgical intervention. }{ }^{53} \text { Higher } \\
\text { incidence in patients with a history of thyroid disorders }{ }^{53}\end{array}$ \\
\hline \multicolumn{3}{|l|}{ Other Disorders } \\
\hline Infusion-associated reactions & $>90 \%$ (serious, $3 \%)^{38,42}$ & $\begin{array}{l}\text { Occurred within } 24 \text { hours of alemtuzumab administration. Mostly mild to moderate; } \\
\text { rarely led to treatment discontinuation. May be caused by cytokine release } \\
\text { following monoclonal antibody-mediated cell lysis }\end{array}$ \\
\hline Infections & $71 \%$ (serious, $2.7 \%)^{53}$ & $\begin{array}{l}\text { Incidence highest during first month after infusion; rate decreased over time. } \\
\text { Mostly mild to moderate in severity. Generally of typical duration; resolved following } \\
\text { conventional medical treatment }\end{array}$ \\
\hline
\end{tabular}

\section{Case Example 2 - Second-line treatment}

A 47-year-old female with MS who had received IFN $\beta$-1b for over 12 months had a brain stem relapse with diplopia. She was started on an induction scheme with mitoxantrone due to perceived bad prognosis. Despite receiving three to four courses, she relapsed again with right arm numbness and urinary urge incontinence and later on she developed paresis of the right foot, increasing her EDSS from 0 to 3.0. She was given two courses of alemtuzumab after which she had no more relapses, MRI scans were stable and her EDSS remained at 3.0.

\section{Case Example 3 - Third-line treatment}

A 52-year-old female patient responded well to IFN $\beta$-1b for almost 1 year, but then had a relapse with a new MRI lesion and so treatment was switched to natalizumab. At the end of the first year of this treatment she had three new relapses over 3-4 months. She tested negative for anti-natalizumab antibodies. She was therefore given two courses of alemtuzumab. Her condition improved and she remained stable without further relapses for the next 3 years.

These cases indicate that alemtuzumab is applicable as first-, secondand third-line therapies in MS and provides disease control even in difficult cases that are refractory to other treatments.

There is a large body of evidence supporting the use of alemtuzumab in RRMS, but little on its use in progressive MS. Studies from the Cambridge cohort $(n=22)$ have assessed treatment responses for a duration of more than 10 years. The relapse rate was significantly reduced in both RRMS and SPMS cohorts. Relapse reduction was $91 \%$ in RRMS patients, from 2.21/patient/year before treatment to 0.19 / patient/year after treatment $(p<0.0001)$. Among the SPMS patients, the relapse rate fell from $0.7 /$ patient/year to $0.01 /$ patient/year $(p<0.001)$, which translates to a $98.6 \%$ reduction. ${ }^{51,52}$ However, the small number of patients and the natural history of SPMS make it difficult to draw definite conclusions about the role of alemtuzumab in progressive MS. These studies also showed that longer-term disability benefits are greater in patients with early RRMS. Some patients whose disability progression was initially controlled, later worsened over the following 10-year period. In this cohort, however, some patients received only one course of alemtuzumab, which may have limited its long-term efficacy. These results emphasise the principle that there is a 'window of opportunity' in early RRMS in which neurological damage can be much more effectively inhibited than during advanced disease. Treatment at later stages of MS can help stabilise disability progression and relapses to a lesser extent and more effort is needed to better understand and support such interventions.

Alemtuzumab treatment has a favourable safety profile but like many high-efficacy treatments in MS, the clinical trial programme identified some risks that need to be managed (see Table 2). Compared with IFN $\beta$ 1a s.c., alemtuzumab raises the risk of certain infections ( $71 \%$ versus $53 \%$ ). ${ }^{53}$ These include urinary tract infection, upper respiratory tract infection, sinusitis, oral herpes, herpes zoster, bronchitis, pharyngitis and gastroenteritis. The infections do not correlate with lymphocyte counts and are predominately mild to moderate, generally of typical duration and resolve following conventional treatment. ${ }^{53,54}$ Acyclovir prophylaxis during and for a month after treatment is recommended to prevent herpes infections.

Infusion-associated reactions are common with alemtuzumab administration, occurring in over $90 \%$ of patients. ${ }^{53}$ These arise within 24 hours of administration and are mostly mild to moderate and rarely lead to treatment discontinuation. Prophylaxis with methylprednisolone, antihistamines and antipyretics can minimise these reactions.

Alemtuzumab is also associated with increased incidence of autoimmune conditions including immune thrombocytopenic purpura (ITP), nephropathies and mainly autoimmune thyroid disorders. About $36 \%$ of patients developed either hypo- or hyper-thyroid disorders during the first 48 months after first alemtuzumab exposure. ${ }^{55}$ The incidence of these disorders peaked at year 3 and declined at year 4 in both the CAMMS223 and CARE-MS I and II trials. ITP is a rare AE occurring in approximately $1 \%$ of patients. It can occur without warning after successive doses of alemtuzumab. Long-term follow-up of the phase II and III studies showed that monitoring was effective in early detection of all cases of ITP, with decreasing incidence from year 
1 to year 3 and no cases in years 4 and $5 .{ }^{56}$ In clinical studies, $0.3 \%$ of alemtuzumab-treated patients developed thyroid cancer and two cases of thyroid cancer were diagnosed in alemtuzumab-treated patients during observational studies. ${ }^{57}$ It is not currently known if alemtuzumab confers a higher risk for developing thyroid malignancies..$^{56}$ Melanoma in $0.3 \%$ and rare cases of lymphoproliferative disorders and lymphoma have also been reported in patients treated with alemtuzumab. ${ }^{57}$

The use of alemtuzumab, therefore, requires appropriate patient selection and risk-management programmes. Before alemtuzumab is given, patients need to be educated on the risks and benefits of the treatment, as well as on pregnancy issues where appropriate. They should also be evaluated for active or latent tuberculosis (TB), screened for hepatitis B and C virus infections where appropriate, tested for complete blood count (CBC), serum creatinine, urinalysis, thyroid function tests and antibody status against varicella-zoster virus (VZV) to determine the need for anti-VZV vaccination. Immediately prior to alemtuzumab administration, pre-treatment with corticosteroids for 3 to 5 days of treatment, anti-histamines and antipyretics to reduce infusion-associated reactions and acyclovir from the first day of treatment and continuing for a minimum of 1 month following treatment are recommended. Treatment schemes can be adapted depending on the setting (in day-care/outpatient clinic or in hospital). Patient education and surveillance should also continue with monitoring activities during treatment for 48 months after the last dose, which include monthly CBC with differential, serum creatinine and urinalysis, and thyroid function tests every 3 months. ${ }^{53}$

Together, the clinical study data from the three major trials, the patient examples given above and the accumulated safety experience demonstrate the efficacy of alemtuzumab and support a favourable benefit-risk profile in patients with active RRMS.

\section{Conclusion}

Both teriflunomide and alemtuzumab have shown efficacy with favourable tolerability and safety profiles in extensive and large-scale clinical trials in MS. Both of these drugs have demonstrated an ability to stabilise disability progression and in many cases even to improve disability. As with other MS treatments, however, early intervention is vital to prevent or reduce irreversible damage. These drugs constitute significant additions to the therapeutic options available for MS. As an oral treatment, teriflunomide is likely to be used mainly in RRMS in early and/or active disease as illustrated in the case examples. Oral treatments in MS are more acceptable to many patients than frequent injections and therefore likely to improve adherence. The three alemtuzumab-treated cases demonstrate that although this treatment may be mainly reserved for more active disease, it is versatile and can be used effectively as a first-, second- or third-line therapy. The dosing courses at 12-month intervals and the long-lasting effect are advantageous to both clinicians and patients, reducing the need for further DMTs.

Further data on the long-term benefits of alemtuzumab on disability progression are needed. The Cambridge cohort has provided some insights over 10 years but on a small sample with variable dosing. The value of alemtuzumab in SPMS requires further investigation; the CARE-MS trial extension and longer term use in the real world will provide further insights into this aspect of its use and help establish its place in treatment algorithms and guidelines.

Teriflunomide has a favourable tolerability and safety profile, the treatment requires only routine monitoring and is easy to manage in clinical practice. Alemtuzumab is associated with more frequent AEs such as infusion-associated reactions, secondary autoimmunity and infections. These, however, can be managed with prophylactic and symptomatic treatment and detected early by careful monitoring and should not be a barrier to use in most MS cases. The licensing of both drugs for use in Europe and the US and their approval for use in RRMS by the UK National Institute for Healthcare and Clinical Excellence (NICE) is likely to increase their use and change the treatment experience and outcomes for many patients with MS. Further data on long-term use of these drugs both from clinical trials and real-world use are awaited with interest.
1. Merck-Serono Europe Ltd, Summary of product characteristics - Rebif solution for injection in cartridges, 2014. Available at: www.medicines.org.uk/emc/ medicine/22368 (accessed 9 April 2015)

2. Biogen Idec Ltd, Summary of product characterstics Tecfidera 240 mg gastro-resistant hard capsules, 2014. Available at: www.medicines.org.uk/emc/medicine/28592 (accessed 9 April 2015)

3. Derfuss T, Personalized medicine in multiple sclerosis: hope or reality? BMC Med, 2012:10:116.

4. Devonshire V, Lapierre Y, Macdonell R, et al., The Global Adherence Project (GAP): a multicenter observational stud on adherence to disease-modifying therapies in patients with relapsing-remitting multiple sclerosis, Eur J Neurol, 2011;18:69-77.

5. FoX EJ, Rhoades RW, New treatments and treatment goals for patients with relapsing-remitting multiple sclerosis, Curr Opin Neurol, 2012; Suppl. 25:S11-9.

6. Johnson FR, Van Houtven G, Ozdemir S, et al., Multiple sclerosis patients' benefit-risk preferences: serious adverse event risks versus treatment efficacy, J Neurol, 2009;256:554-62.

7. Lobentanz IS, Asenbaum S, Vass K, et al., Factors influencing quality of life in multiple sclerosis patients: disability, depressive mood, fatigue and sleep quality, Acta Neuro depressive mood, fatig

8. Lorefice L, Mura G, Coni G, et al., What do multiple sclerosis patients and their caregivers perceive as unmet needs? BMC Neurol, 2013;13:177.

9. Miller A, Avidan N, Tzunz-Henig N, et al., Translation towards personalized medicine in Multiple Sclerosis, J Neurol Sci, 2008;274:68-75

10. Patti F, Optimizing the benefit of multiple sclerosis therapy: the importance of treatment adherence, Patient Prefer Adherence, 2010;4:1-9.

11. Tanasescu R, Ionete C, Chou IJ, et al., Advances in the treatment of relapsing-remitting multiple sclerosis, Biomed J 2014:37:41-9.

12. Haghikia A, Gold R, Multiple sclerosis: TOWER confirms the efficacy of oral teriflunomide in MS, Nat Rev Neurol, 2014;10:183-4.

13. Bayas $\mathrm{A}$, Maurer $\mathrm{M}$, Teriflunomide for the treatment of relapsing-remitting multiple sclerosis: patient preference and adherence, Patient Prefer Adherence, 2015;9:265-4.

14. Oh $\mathrm{J}, \mathrm{O}^{\prime} \mathrm{C}$ (nnnor PW, Teriflunomide in the treatment of multiple sclerosis: current evidence and future prospects, Ther Adv Neurol Disord, 2014:7:239-52.

15. Confavreux C, Li DK, Freedman MS, et al., Long-term followup of a phase 2 study of oral teriflunomide in relapsing multiple sclerosis: safety and efficacy results up to 8.5 years, Mult Scler, 2012;18:1278-89.

16. O'Connor P, Wolinsky JS, Confavreux C, et al., Randomized trial of oral teriflunomide for relapsing multiple sclerosis, N Engl J Med, 2011;365:1293-303

17. Confavreux C, O'Connor P, Comi G, et al., Oral teriflunomide for patients with relapsing multiple sclerosis (TOWER): a randomised, double-blind, placebo-controlled, phase 3 trial, Lancet Neurol, 2014;13:247-56.

18. Vermersch P, Czlonkowska A, Grimaldi LM, et al., Teriflunomide versus subcutaneous interferon beta-1a in patients with relapsing multiple sclerosis: a randomised, controlled phase 3 trial, Mult Scler, 2014:20:705-16.

19. Miller AE, Wolinsky JS, Kappos $L$, et al., Oral teriflunomide for patients with a first clinical episode suggestive of multiple sclerosis (TOPIC): a randomised, double-blind, placebocontrolled, phase 3 trial, Lancet Neurol, 2014;13:977-86.

20. Bar-Or A, Freedman MS, Kremenchutzky M, et al., Teriflunomide effect on immune response to influenza vaccine in patients with multiple sclerosis, Neurology, 2013;81:552-8.

21. Freedman F, Confavreux C, Olsson T, et al., 2013, Teriflunomide Efficacy and Safety Analyses: Results From TEMSO and TOWER (P5), Americas Commitee on treatment and Research in Multiple Sclerosis (ACTRIMS) Orlando, Florida, US.

22. Wolinsky JS, Narayana PA, Nelson F, et al., Magnetic resonance imaging outcomes from a phase III trial of teriflunomide, Mult Scler, 2013:19:1310-9.

23. Bar-Or A, 2013, Immune response to neoantigen and recall antigens in healthy subjects receiving teriflunomide P622, 29th Congress of the European Committee on Treatment and research in Multiple Sclerosis (ECTRIMS) Copenhagen, Denmark.

24. Menguy-Vacheron F, Varona R, Posevitz-Fejfar A, et al., 2013, Exploring the impact of teriflunomide on immune cells in patients with relapsing multiple sclerosis: design of the Teri-Dynamic study, 23rd Meeting of the European Society of Neurology, Barcelona, Spain.

25. Vollmer TL, Stalla-Bourdillon A, Robinson M, 2013, Teriflunomide in Routine Clinical Practice: Design of the TeriPRO Study DX58, 5th Cooperative Meeting of the Consortium of Multiple Sclerosis Centers (CMSC), Orlando, Florida, US.

26. Chitnis T, Banwell B, Arnold DL, et al., 2014, Study Design of a Phase 3 Trial Evaluating Teriflunomide in Children and Adolescents with Relapsing Multiple Sclerosis, Cooperative Meeting of the Consortium of Multiple Sclerosis Centers, Dallas, Texas, US.

27. Kappos L, Comi G, Freedman MS, et al., 2013, Pooled efficacy data from two phase 3 placebo-controlled trials of oral, oncedaily teriflunomide P618, 29th Congress of the European Ciommittee for Treatment and Research in Multiple Sclerosis, Copenhagen, Denmark.

28. Freedman MS, Dukovic D, Benamor M, et al., 2014, Consistent treatment effect of teriflunomide in subgroups based on pretrial therapy: pooled analyses of TEMSO and TOWER (DX47) Annual Meeting of the Consortium of Multiple Sclerosis Centers (CMSC), Dallas, Texas, USA

29. Macdonell $R, 2013$, Teriflunomide reduces relapse-related sequelae, severe relapses, hospitalisations and corticosteroid use: pooled data from the phase 3 TEMSO and TOWER studies (P1095). 29th Congress of the European Committee for Treatments and Research in Multiple Sclerosis, Copenhagen, Denmark

30. Marurer M, 2013, Impact of severe relapses on disability, fatigue and healthrelated quality of life outcomes: a pooled dataset of the phase 3 TEMSO and TOWER studies, 29th congress of the European Committee on Treatment and 
Research in Multiple Sclerosis, Copenhagen, Denmark.

31. Freedman MS, Comi G, Kappos L, et al., 2013, Long-term safety and efficacy of teriflunomide in patients with relapsing forms of multiple sclerosis in the TEMSO extension trial (P544), 29th Congress of the European Committe for Treatment and Research in Multiple Sclerosis Copenhagen, Denmark.

32. Leist T, Hunter SF, Kantor D, et al., Novel therapeutics in multiple sclerosis management: clinical applications, Am J Med, 2014:127:S2.

33. Singer B, Comi G, Miller A, et al., 2013, Frequency of infections during treatment with teriflunomide: pooled data from three during treatment with teriflunomide: pooled data from three Academy of Neurology Annual Meeting, San Diego, CA, USA.

34. sanofi-aventis groupe, Summary of product characteristics AUBAGIO 14 mg film-coated tablets, 2014. Available at: http:// www.medicines.org.uk/emc/medicine/28533 (accessed 6 July 2015).

35. Atkinson MJ, Sinha A, Hass SL, et al., Validation of a general measure of treatment satisfaction, the Treatment Satisfaction Questionnaire for Medication (TSQM), using a national panel study of chronic disease, Health Qual Life Outcomes, 2004;2:12.

36. Vermersch P, CZlonkowska A, Grimaldi L, et al., 2012, Evaluation of patient satisfaction from the TENERE study: a comparison of teriflunomide and subcutaneous interferon beta-1a in patients with relapsing multiple sclerosis, 22nd Meeting of the European Neurologiocal Society, Prague, Czech Republic.

37. Hu Y Turner MJ, Shields J, et al., Investigation of the mechanism of action of alemtuzumab in a human CD52 transgenic mouse model, Immunology, 2009;128:260-70

38. Coles AJ, Twyman CL, Arnold DL, et al., Alemtuzumab for patients with relapsing multiple sclerosis after diseasemodifying therapy: a randomised controlled phase 3 trial, Lancet, 2012;380:1829-39.

39. Cox AL, Thompson SA, Jones JL, et al., Lymphocyte homeostasis following therapeutic lymphocyte depletion in multiple sclerosis, Eur J Immunol, 2005;35:3332-42.

40. Hartung HP, Arnold DL, Cohen JA, 2012, Lymphocyte subset dynamics following alemtuzumab treatment in the CARE-MS 1 study (P 935), 28th Congress of the European Committee for Treatment and Research in Multiple Sclerosis (ECTRIMS), Lyon, France.

41. Turner MJ, Lamorte MJ, Chretien $\mathrm{N}$, et al., Immune status following alemtuzumab treatment in human CD52 transgenic mice, I Neuroimmunol, 2013;261:29-36.
42. Cohen JA, Coles AJ, Arnold DL, et al., Alemtuzumab versus interferon beta $1 \mathrm{a}$ as first-line treatment for patients with relapsing-remitting multiple sclerosis: a randomised controlled phase 3 trial, Lancet, 2012;380:1819-28.

43. CAMMS223 Trial Investigators, Coles AJ, Compston DA, et al., Alemtuzumab vs. interferon beta-1a in early multiple sclerosis, N Engl J Med, 2008;359:1786-801.

44. Arroyo R, Arnold DL, Cohen J, et al., 2013, Alemtuzumab improves quality of life compared to SC IFNB-1a in CARE-MS II (P531), 23rd Meeting of the European Neurological Society, Barcelona, Spain. Available at: www.congrex-switzerland. Barcelona, Spain. Available at: WwW.congrex-switzerland. (accessed 6 July 2015).

45. Gupta A, Arnold D, Cohen J, et al., 2012, Alemtuzumab improves quality of life compared to SC IFNB-1A in CARE-MS I, Annukla meeting of the Consortium Multiple Sclerosis Centers, San Diego, CA, US.

46. Coles AJ, 2014, Efficacy and safety of alemtuzumab in treatment naive patients with relapsing-remitting MS: fouryear follow-up of the CARE-MS I study (P090) Joint Meeting of the American Committe for Treatment and Research in Multiple Sclerosis and European Committe for Treatment and Research in Multiple Sclerosis, Boston, Massachusetts, US,

47. Hartung HP, Arnold DL, Cohen JA, 2014, Efficacy and safety of alemtuzumab in patients with relapsing-remitting MS who relapsed MS II study (P043), 201 (Suppl), Joint Meeting of the American Committe for Treatment and Research in Multiple Sclerosis and European Committe for Treatment and Research in Multiple Sclerosis, Boston, MA, US.

48. Arnold DL, 2014, Alemtuzumab improves brain MRI outcomes in patients with active relapsing-remitting multiple sclerosis: three-year followup of the CARE-MS studies (P008), 66th Annual Meeting of the Amerian Academy of Neurology, Philadelphia, PA, US

49. Arnold D, Brinar V, Cohen J, 2012, Effect of alemtuzumab vs Rebif $\Theta$ on brain MRI measurements: results of CARE-MS I, a phase 3 study (S11.006), Annual Meeting of the American Academy of Neurology, New Orleans, LA, USA.

50. Arnold DL, 2012, Effect of alemtuzumab vs Rebif $\circledast$ on brain MRI measurements (P877), European Committee on

Coles AJ, Cox A, Le Page E, et al., The window of therapeutic opportunity in multiple sclerosis: evidence from monoclonal antibody therapy, I Neurol, 2006;253:98-108.
52. Coles AJ, Wing MG, Molyneux P, et al., Monoclonal antibody treatment exposes three mechanisms underlying the clinical course of multiple sclerosis, Ann Neurol, 1999;46:296-304.

53. Genzyme Therapeutics Ltd, Summary of product characteristics - LEMTRADA $12 \mathrm{mg}$ concentrate for solution for infusion, 2013. Available at: www.medicines.org.uk/emc/ medicine/28917 (accessed 14 April 2015)

54. Havrdova E, 2013, Infection risk with alemtuzumab in patients with relapsing remitting multiple sclerosis: pooled results from the CARE-MS I and CARE-MS II trials, 29th Congress of the European Committee on treatment and Research in of the European Committee on treatment

55. Twyman C, Oyuela P, Palmer J, et al., Thyroid autoimmune adverse events in patients treated with alemtuzumab for relapsing-remitting multiple sclerosis: four-year follow-up of the CARE-MS studies, Neurology, 2014;82 Supplement P2.199.

56. Cuker A, Stasi R, Palmer J, et al., Successful detection and management of immune thrombocytopenia in alemtuzumabtreated patients with active relapsing-remitting multiple sclerosis, Neurology, 2014;82 10 Supplement:1526

57. Babij R, Perumal JS, Comparative efficacy of alemtuzumab and established treatment in the management of mu . Neuropsychiatr Dis Treat, 2015:11:12

58. McDonald WI, Compston A, Edan G, et al., Recommended diagnostic criteria for multiple sclerosis: guidelines from the International Panel on the diagnosis of multiple sclerosis, Ann Neurol, 2001:50:121-7.

59. Polman CH, Reingold SC, Edan G, et al., Diagnostic criteria for multiple sclerosis: 2005 revisions to the "McDonald Criteria", Ann Neurol, 2005;58:840-6.

60. Wynn D, Arnold DL, Coles AJ, Detection, incidence, and management of glomerulonephritis in the alemtuzumab clinical development program (P597) 29th Congress of the European Committee for Research and Treatment in Multiple Sclerosis (ECTRIMS), Copenhagen, Denmark.

61. Coles AJ, Fox E, Vladic A, et al., Alemtuzumab more effective than interferon beta-1a at 5-year follow-up of CAMMS223 clinical trial, Neurology, 2012;78:1069-78.

62. Cohen J, Twyman C, Arnold D, et al., 2012, Efficacy and safety results from comparison of alemtuzumab and Rebif ${ }^{\oplus}$ efficacy in multiple sclerosis II (CARE-MS II): A phase 3 study in relapsing-remitting multiple sclerosis patients whorelapsed on Prior therapy (S01.004), Annual Meeting of the American Academy of Neurology, New Orleans, LA, US. 